\title{
Erratum to: A 30-year epidemiologic survey (1974-2003) of haematological malignancies on the island of Sardinia: temporal changes in incidence and a geographic comparison of incidence rates
}

\author{
G. Broccia • M. Longinotti • A. Gabbas • Maria Monne • \\ Caterina Porcu $\cdot$ E. Chessa $\cdot$ Barbara Giannico
}

Published online: 21 February 2014

(C) Springer-Verlag Berlin Heidelberg 2014

\section{Erratum to: Ann Hematol}

DOI 10.1007/s00277-013-2002-z

The original version of this article inadvertently contained a mistake. Maria Monne, who also contributed to the study, was not included in the author list.

\footnotetext{
The online version of the original article can be found at http://dx.doi.org/ 10.1007/s00277-013-2002-z.

G. Broccia $(\bowtie)$

Divisione di Ematologia e Centro Trapianti Midollo osseo, Ospedale Oncologico A. Businco, via Jenner 9, 09121 Cagliari, Italy

e-mail: giorgio.broc@tiscali.it

M. Longinotti $\cdot$ B. Giannico

Istituto di Ematologia, Università di Sassari, Viale San Pietro,

07100 Sassari, Italy

A. Gabbas $\cdot$ M. Monne

Divisione di Ematologia, Ospedale San Francesco, Via Mannironi, 08100 Nuoro, Italy

C. Porcu

Divisione di Medicina, Ospedale San Francesco, Via Mannironi,

08100 Nuoro, Italy

E. Chessa

Divisione di Medicina, Ospedale San Martino, Via Rockfeller, 09170 Oristano, Italy

Present Address:

G. Broccia

Via Guzzoni degli Ancarani 6, 09121 Cagliari, Italy
} 